\title{
le phénomène d'effondrement dans les roches volcaniques à forte porosité
}

\author{
par \\ Santiago Uriel Romero \\ Chef du Département de Géotechnique \\ Laboratorio de Carreteras y Geotecnia "José Luis Escario", Madrid \\ Vice-président pour l'Europe \\ Société Internationale de Mécanique des roches
}

\section{Introduction}

On dit qu'il y a "effondrement " d'un sol ou d'une roche quand la modification d'une condition externe quelconque produit un changement substantiel dans sa structure, avec une réduction de volume plus ou moins importante.

Diverses conditions externes provoquent ces changements. Dans le cas du sable lâche, un effort dynamique peut provoquer l'effondrement d'une structure très peu stable. Parfois ce sont les changements de teneur en eau qui sont à l'origine de cette réduction de volume, particulièrement dans les argiles gypsifères, les lœss ou les sols semi-saturés mal compactés. La troisième cause d'effondrement tient aux seules contraintes statiques, indépendamment de variations de teneur en eau du matériau. Dans ce cas se trouvent quelques limons gypsifères, certains sols sensibles cimentés, et les roches à forte porosité.

Celles-ci sont le sujet des études exposées ci-dessous.

Parmi toutes les roches poreuses, ce sont sans doute celles d'origine volcanique qui présentent le plus clairement le phénomène d'effondrement. II est évident que le degré d'effondrement général dépend de la facilité qu'a la structure naturelle de la roche de devenir plus compacte, et c'est pour cela que le phénomène se manifeste de plus en plus nettement lorsque la densité de la roche diminue. Dans les roches compactes, le phénomène d'effondrement ne peut se manifester que d'une manière limitée.

Les roches volcaniques formées par l'agglomération de cendres et de pyroclastes émis par des volcans et sédimentés à l'air ont une structure et une composition très différentes selon le matériau émis et selon le degré de cimentation entre les fragments, cendres ou pyroclastes, en fonction de la température et des conditions de contraintes pendant la soudure.

Le sujet est subdivisé en cinq parties:

- un rappel de la composition minéralogique et de la structure de ces roches;
- leur comportement mécanique déduit des essais in situ et au laboratoire;

- la formulation d'une théorie qui explique le phénomène d'effondrement à partir de l'expérimentation précédente:

- un résumé des propriétés géotechniques les plus importantes, pour juger de leur aptitude à supporter des fondations;

- enfin quelques exemples d'ouvrages et de problèmes posés dans ce type de roches volcaniques.

\section{Nature et structure des roches voicaniques}

Les résultats présentés correspondent à des études réalisées pour quatre ouvrages importants dans les lles Canaries :

a) Fondation du pont "Cuesta de Silva ". La roche est un agglomérat très hétérométrique, avec d'abondants xénolithes de phonolithe englobés dans une matrice micro-agglomératique (un tuf) de couleur crème, bistre, ou occasionnellement grise. Ce matériau n'a pas de stratification apparente, il se présente en forme de masses chaotiques, avec un degré de lithification variable.

b) Barrage de "Los Campitos ". L'agglomérat de Los Campitos est constitué dans sa majorité par des tufs hybrides altérés de lapilli et de cinérites, avec une matrice sablo-argileuse, le matériau pyroclastique est du type basalte spongieux. Dans la fraction argileuse, il y a abondance de minéraux mal cristallisés, généralement du type kaolin, principalement des halloysites, avec un peu de montmorilIonite.

c) Barrage “Ariñez ». L'agglomérat d'Ariñez est constitué par des fragments de téphrite inclus dans une importante masse de microcristaux de pyroxènes, plagioclases et feldspaths, avec un ciment de métahalloysite et montmorillonite, ainsi que d'autres minéraux argileux en proportion inférieure à $4 \%$, 
d) “Punta Camello », L'agglomérat de Punta Camello est constitué par des ponces et des cendres avec un liant dont les caractéristiques sont très variées. Les matériaux déposés et les altérations postérieures conduisent à des compositions et des structures très variables. Les figures 1 à 5 montrent certaines de ces structures, vues au microscope électronique à balayage. Toutes correspondent à des agglomérats de Punta Camello et donnent une idée claire de la diversité mentionnée ci-dessus.

Sur la figure 1 ( $\times 3900)$ les fragments ponceux laissent de grands vides et les liaisons entre eux sont courtes. Sur la figure 2 ( $\times 780$ ) une structure vacuolaire est entourée par un matériau continu formant une sorte de nid d'abeilles de forme irrégulière. Dans d'autres zones (fig. $3, \times 320$ ) des recristallisations fibreuses de silico-aluminates hydratés se développent dans les vides d'une structure similaire à celle des figures 1 et 2 . Parfois ces fibres recouvrent simplement les parois, et parfois elles servent de liaison entre ces parois. Avec un grossissement plus fort (fig. $4, \times 8000$ ) ces cristallisations sont bien visibles. Quelquefois (fig. 5 , $x 78$ ) ces fibres s'agglomèrent dans les vides et y forment une structure réticulée très irrégulière.

L'analyse à la microsonde électronique met aussi l'accent sur la grande diversité dans la composition de ces roches ponceuses (fig. 6). En général, les pics correspondant à la silice, I'aluminium et au potassium sont les plus prononcés, les autres correspondant aux cations, fer, sodium, magnésium, calcium, titane et soufre étant variables. II s'agit par conséquent de ponces de nature calco-alcaline, riches en silice et alumine, soit d'origine primaire, soit de néo-formation.

La densité apparente sèche varie dans des limites très larges. Les moins denses correspondent à Punta Camello : $0,45 \mathrm{t} / \mathrm{m}^{3}$. Les plus denses peuvent dépasser $1,6 \mathrm{t} / \mathrm{m}^{3}$. Les dépôts sont généralement très irréguliers, en fonction des éruptions et sédimentations successives au cours des temps géologiques. Si les épaisseurs sont importantes, plusieurs dizaines de mètres, et si le dépôt est survenu dans un temps très court, probablement au cours d'une même éruption, la densité croît nettement avec la profondeur. Les roches les plus superficielles ayant une densité de l'ordre de $1,15,20$ mètres plus bas la densité augmente jusqu'à 1,5 ou 1,6. Cela est dû à une consolidation préalable à la soudure ou la cimentation entre les particules qui forment la roche. Mais en général la distribution des densités est variable, elle est très erratiquement dispersée à courte distance, mise à part une tendance à augmenter avec la profondeur valable à grande échelle.

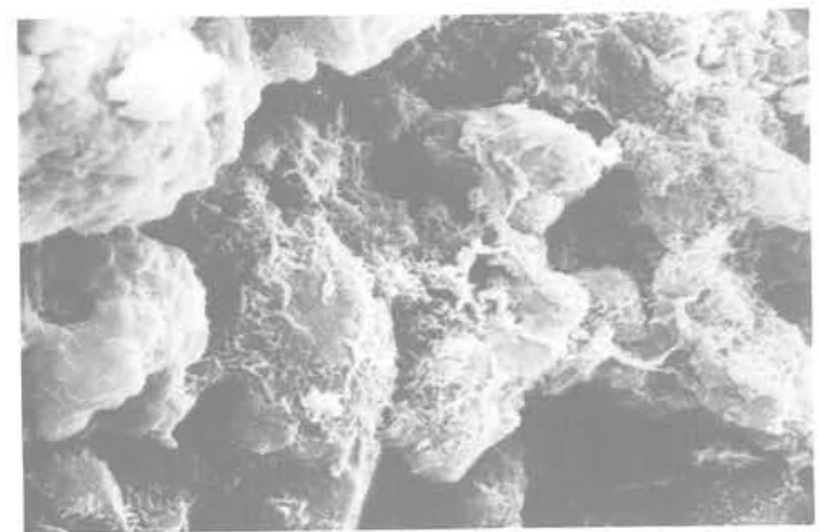

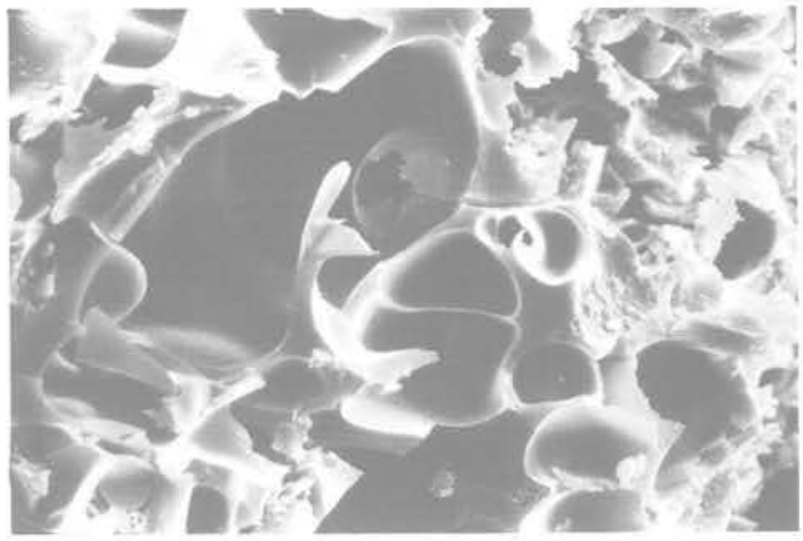
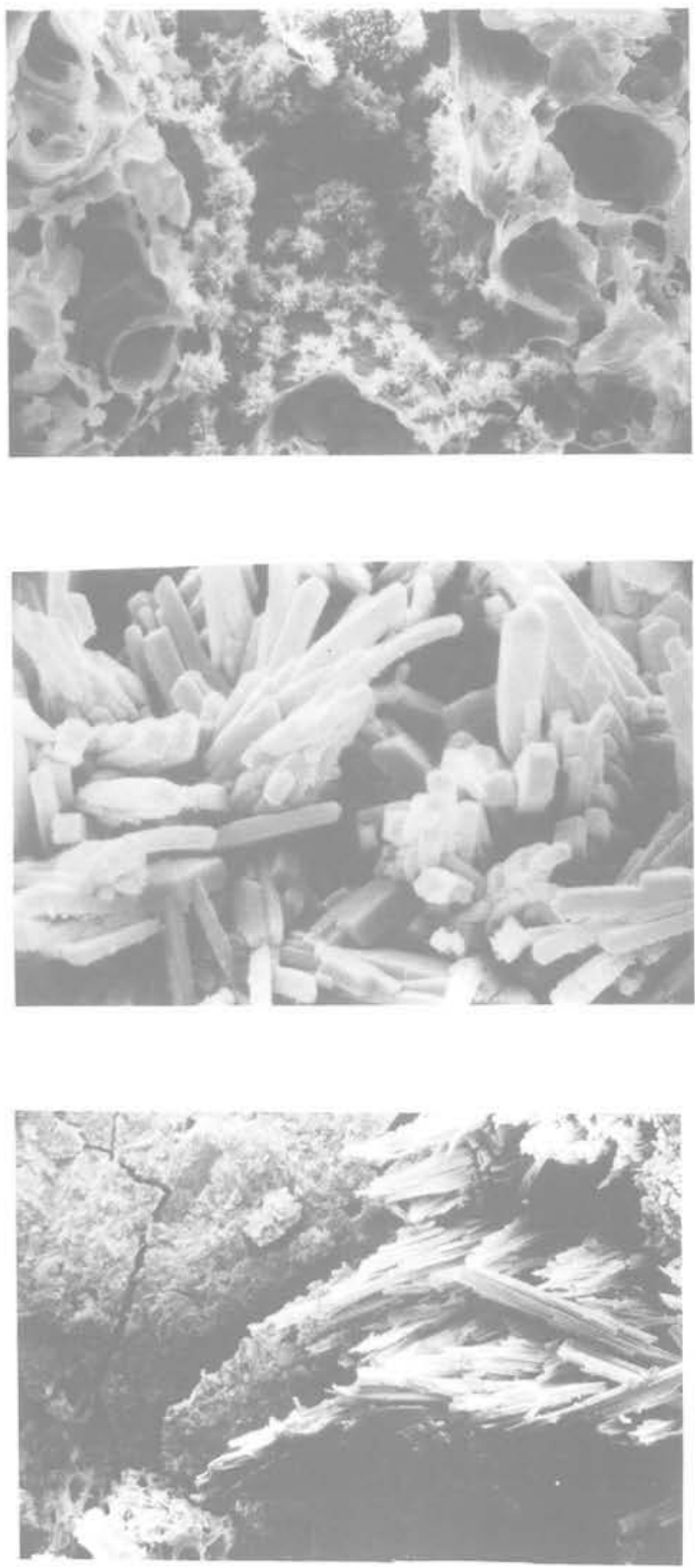

Fig. 1 à 5 Images au microscope électronique à balayage de tufs volcaniques (agglomérats de Punta Camello) 


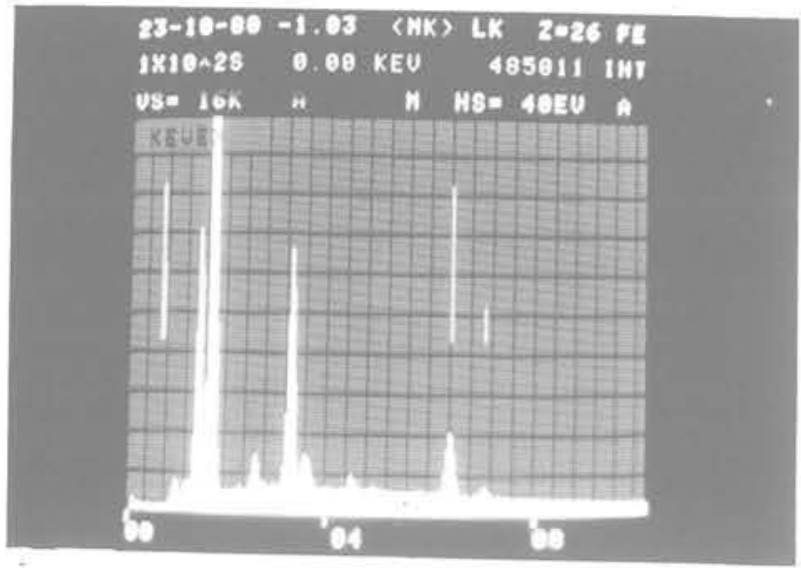

Fig. 6 Diagramme d'analyse à la microsonde électronique

Ces roches volcaniques sont facilement dégradables par tout effort concentré. Leur structure naturelle s'effondre sous des efforts de compression relativement modestes qui dépendent naturellement de leur densité. Elles sont faciles à excaver par tout procédé manuel ou mécanisé.

Généralement, et sauf dans le cas oủ l'altération postgénétique a été importante, leur structure se conserve sans dégradation apparente lorsqu'elles sont immergées dans l'eau. La facilité de taille à la main des variétés les plus légères a permis, dans certaines zones de l'île de Ténériffe, la construction de canaux de petites dimensions faits en éléments assemblés.

Leur perméabilité en masse est basse et généralement les quelques fissures existantes sont peu importantes. Cela est dû probablement au module de déformation relativement bas de ces roches qui permet l'accommodation à n'importe quelle déformation postérieure à leur formation.

C'est seulement dans les plans de liaison des dépôts successifs que la structure du contact peut être perméable. Sauf dans ce dernier cas, un massif de roche volcanique de ce type n'est pas exclu pour la construction de barrages et de retenues. Comme elles ne s'affaissent pas au contact de l'eau et que, sauf cas exceptionnels, leur perméabilité intrinsèque est modérée, il existe des réservoirs souterrains d'eau potable creusés dans ces formations dans certaines zones de l'île de Grande Canarie.

La figure 7 montre le déblai d'une route sur sept mètres de hauteur environ. On remarque quelques strates plus dures en porte-à-faux au-dessus de strates plus sensibles à l'érosion. On voit aussi la trace des dents de la pelle mécanique utilisée pour l'excavation. La figure 8 montre diverses strates de cendres situées en discordance sur une grande masse uniforme de ponces. La figure 9 montre clairement l'érosion différentielle de type éolien. Les strates plus dures dépassent en porte-à-faux, protégeant ainsi les plus tendres de l'érosion.

\section{Comportement triaxial des roches à forte porosité}

Dans les diverses roches citées ci-dessus, le comportement générique est très similaire. Les essais réalisés visent à fixer les conditions de contrainte dans lesquelles se produit la destruction ou la dégradation de la structure naturelle, c'est-à-dire le phénomène d'effondrement.
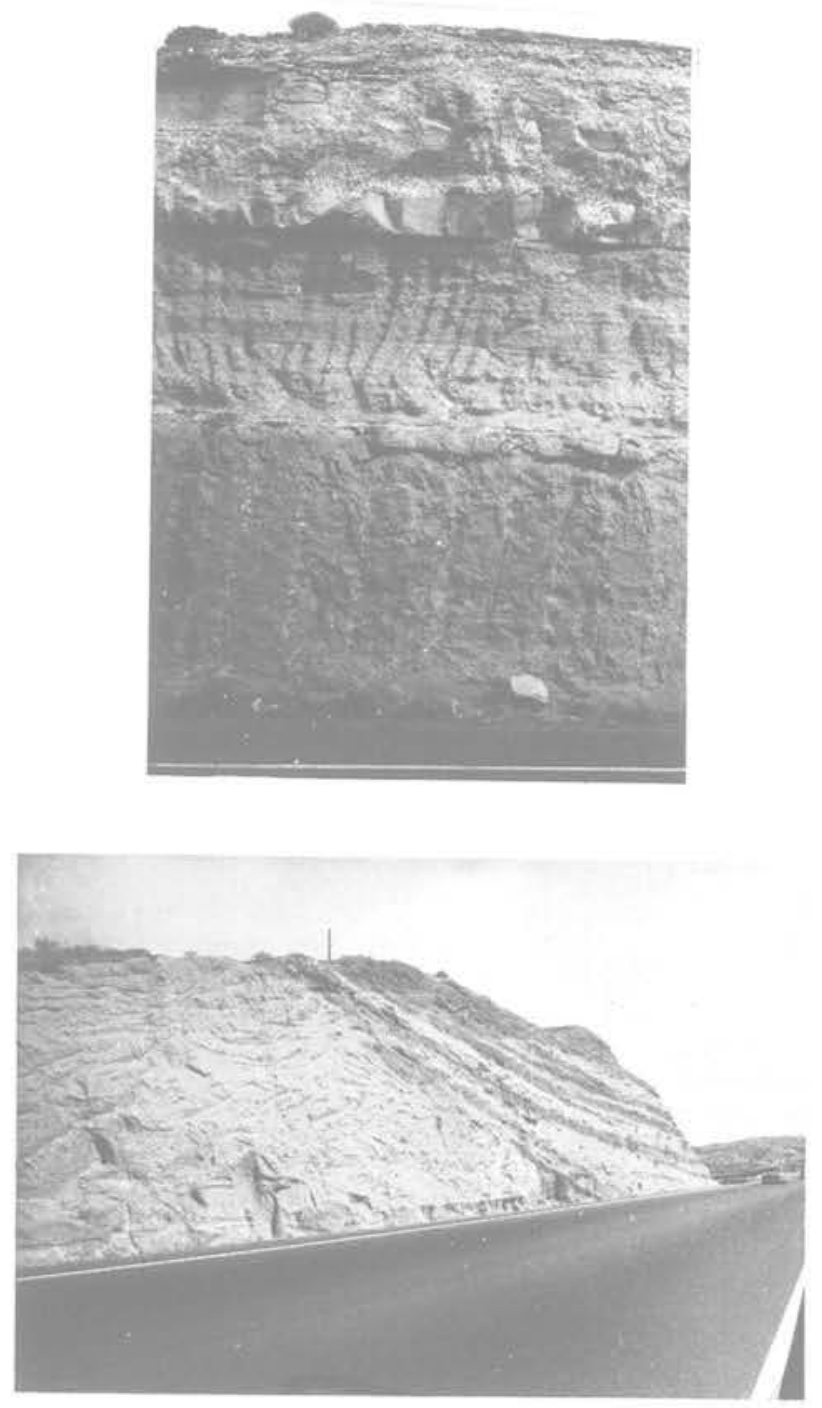

Fig. 7 et 8 Parois de tranchées routières dans I7̂le de Ténériffe, route de Santa Cruz à l'aéroport

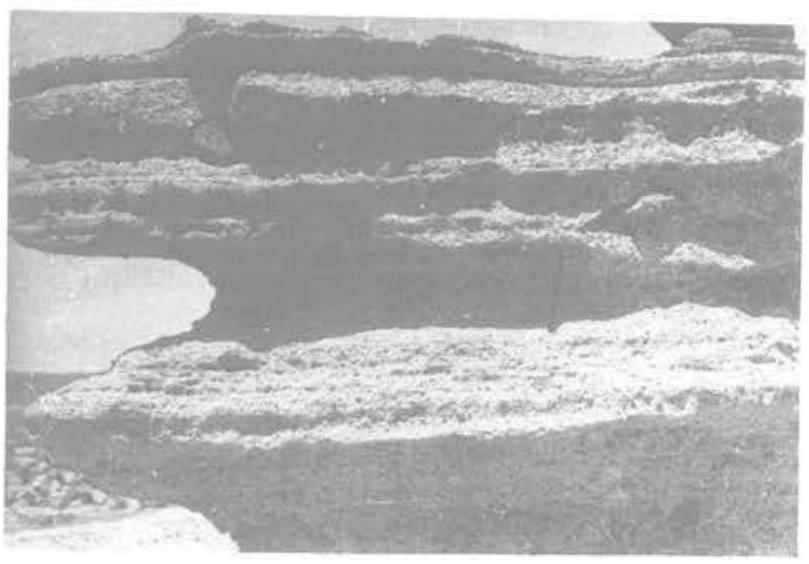

Fig. 9 Falaise naturelle montrant l'érosion différentielle par le vent. Plusieurs bombes sont visibles. Punta Camello

Les essais de laboratoire ont consisté principalement en des essais triaxiaux lents, de manière que les conditions d'humidité aient une faible influence sur les résultats. Deux types d'essais de base ont été effectués.

a) Une compression isotrope croissante.

b) Une consolidation sous pression latérale fixe, suivie d'un accroissement du déviateur. 
La partie inférieure de la figure 10 est une courbe effort-déformation sous pression isotrope. Pour des niveaux de compression inférieurs à une certaine contrainte critique $\sigma_{c}$ (point $\mathrm{M}$ ), le comportement est pratiquement élastique et linéaire. Le module de déformation est élevé, la déformation petite. Lorsqu'on atteint la contrainte $\sigma_{\mathrm{c}}$, il se produit un changement brutal de la déformabilité en raison de la destruction de la structure naturelle de la roche, c'est l'effondrement. Si on augmente davantage la contrainte isotrope, le comportement est encore approximativement linéaire jusqu'à une certaine pression (point $\mathrm{N}$ ) au-dessus de laquelle les déformations diminuent et le module augmente. Cela correspond évidemment à une densification du matériau granulaire dans lequel s'est transformée la roche d'origine après son effondrement.

Si on fait le même essai de compression isotrope sur la même roche préalablement pulvérisée et compactée à la densité de la roche d'origine, on obtient une courbe $\mathrm{Cl}$ qui coïncide sensiblement avec le tronçon final déjà obtenu au-delà du point $\mathrm{N}$.

L'évolution de la structure de la roche pendant les trois étapes indiquées est nette : entre le début de l'essai et la pression $\sigma_{\mathrm{c}}$, le comportement est celui d'une roche élastique; entre les points $M$ et $N$, la roche s'effondre progressivement, toutefois elle conserve un peu de sa structure d'origine; enfin, au-delà du point $N$, les liaisons sont totalement détruites et le comportement correspond à celui d'un sol pulvérulent.

Si on effectue un essai triaxial avec une consolidation isotrope préalable $\sigma_{3}$ inférieure à $\sigma_{c}$, en augmentant le déviateur à partir de cette pression latérale constante, le comportement varie suivant que $\sigma_{3}$ est plus ou moins proche de la valeur critique $\sigma_{c}$ : si $\sigma_{3}$ est proche de $\sigma_{c}$ on obtient le graphique de la figure 11. Lorsque le déviateur augmente, l'effondrement commence pour un cercle de Mohr dont le point le plus haut décrit la ligne CC. La déformation augmente d'une façon linéaire jusqu'au cercle de Mohr dont le point haut est situé sur la courbe FC. Au-delà, on peut considérer que la structure est totalement détruite, le comportement est celui d'un sol granulaire. Ceci est démontré par les essais dans les mêmes conditions de contraintes sur la roche préalablement pulvérisée (ligne DD).

$\mathrm{Si}$ on augmente encore le déviateur, on obtient la rupture finale de ce sol pulvérulent au point $\mathrm{C}$ situé sur la ligne de résistance intrinsèque de ce matériau (ligne SP). La structure de la roche passe par les mêmes étapes successives que dans le cas de la consolidation isotrope.

Par contre si l'essai triaxial est fait avec une valeur petite de $\sigma_{3}$, comme dans le cas de la figure 12 , les résultats sont très différents: la roche se brise de manière fragile, le long d'un ou plusieurs plans de fracture, sans dégradation préalable généralisée. L'effondrement de la structure s'observe seulement dans une bande étroite, à proximité de ces plans, il n'est pas généralisé à l'ensemble du volume.

L'ensemble de ces comportements est donc schématisé à la partie supérieure de la figure 10. L'espace des contraintes est divisé en trois zones A, B, C, limitées par quatre lignes et une ligne de transition. Dans la zone $A$ le comportement est celui d'une roche élastique, dans la zone B le matériau est en cours d'effondrement et dans la zone $\mathrm{C}$ la roche est complètement pulvérisée, le comportement correspond à celui d'un sol granulaire. La ligne CC qui sépare les zones A et B montre le début de l'effondrement; la ligne $\mathrm{FC}$ qui sépare les zones $\mathrm{B}$ et $\mathrm{C}$ en indique la fin.

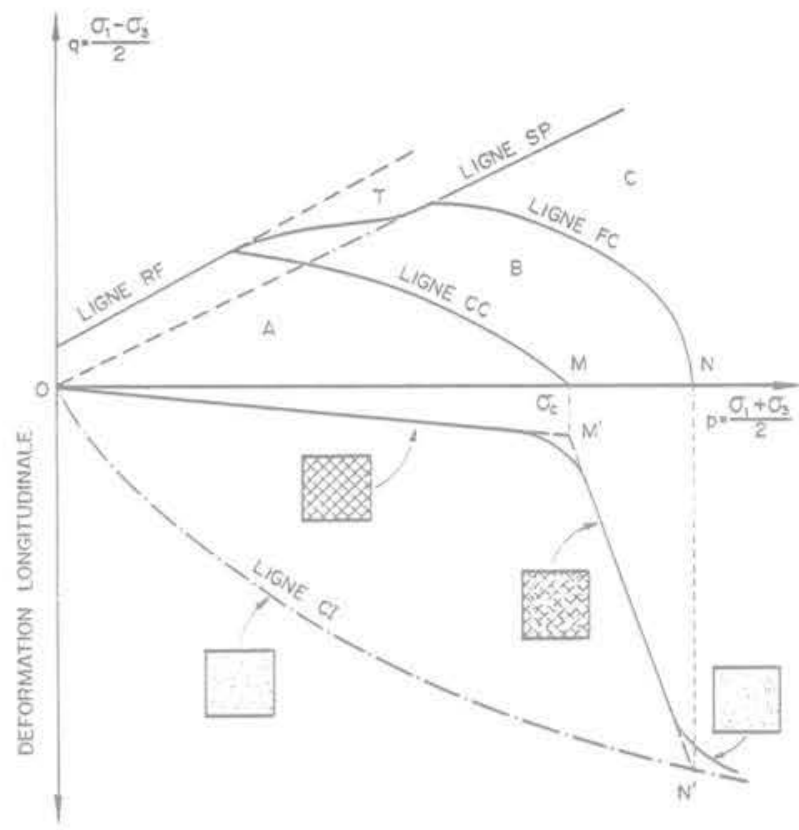

Fig. 10 Essai triaxial isotrope. La ligne Cl est la courbe de consolidation isotrope du matériau préalablement pulvérisé

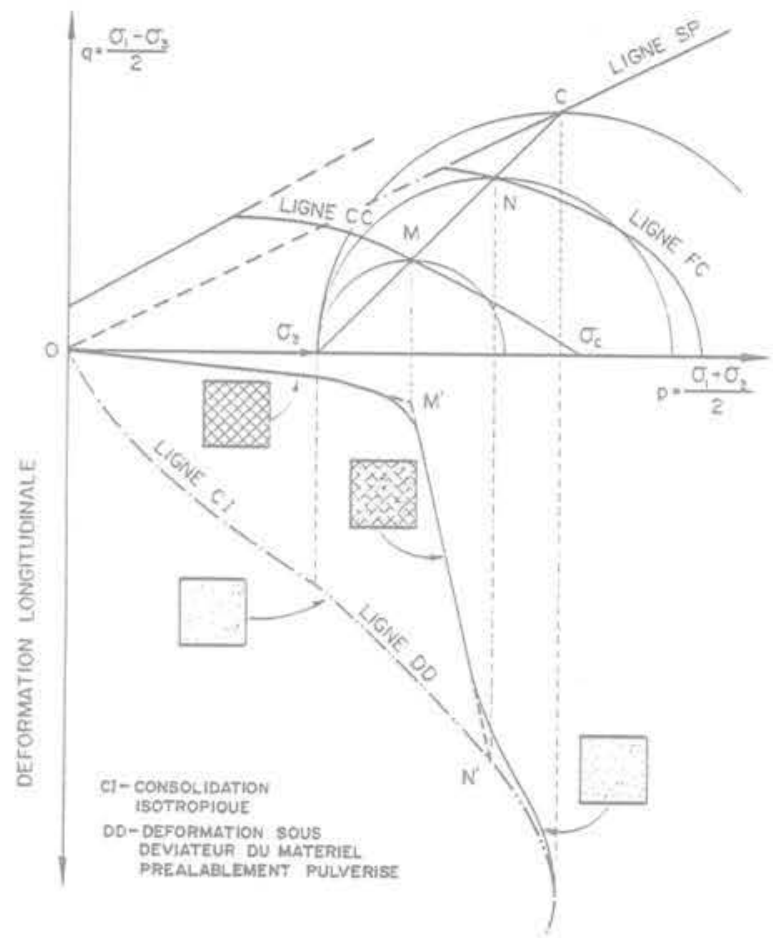

Fig. 11 Essai triaxial avec déviateur, sous forte pression latérale. $\mathrm{Cl}$ est la courbe de consolidation isotrope et $D D$ la courbe de déformation sous déviateur du matériau préalablement pulvérisé

La courbe intrinsèque présente une première partie rectiligne RF. Quand le cercle de Mohr atteint cette ligne, la roche présente une rupture fragile. Pour des contraintes importantes, cette ligne RF se transforme en SP, prolongement de la droite intrinsèque d'un matériau granulaire; entre les deux il y a une courbe $T$ de transition, qui est généralement mal définie par les essais parce que la fracture a lieu dans des conditions hybrides, dans un matériau dont la structure souffre d'une dégradation partielle. 


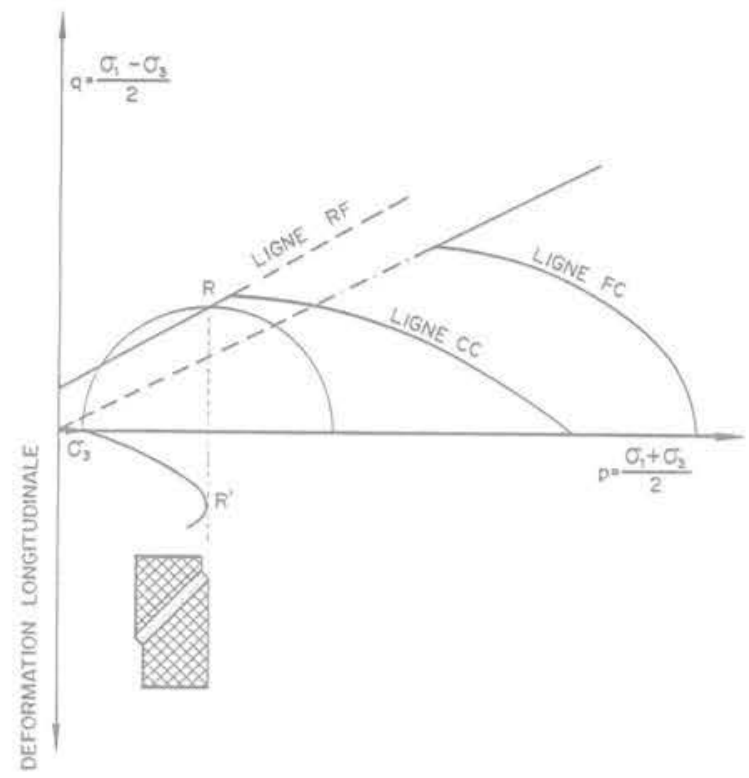

Fig. 12 Essai triaxial avec déviateur, sous faible pression latérale

\section{Structure vacuolaire uniforme}
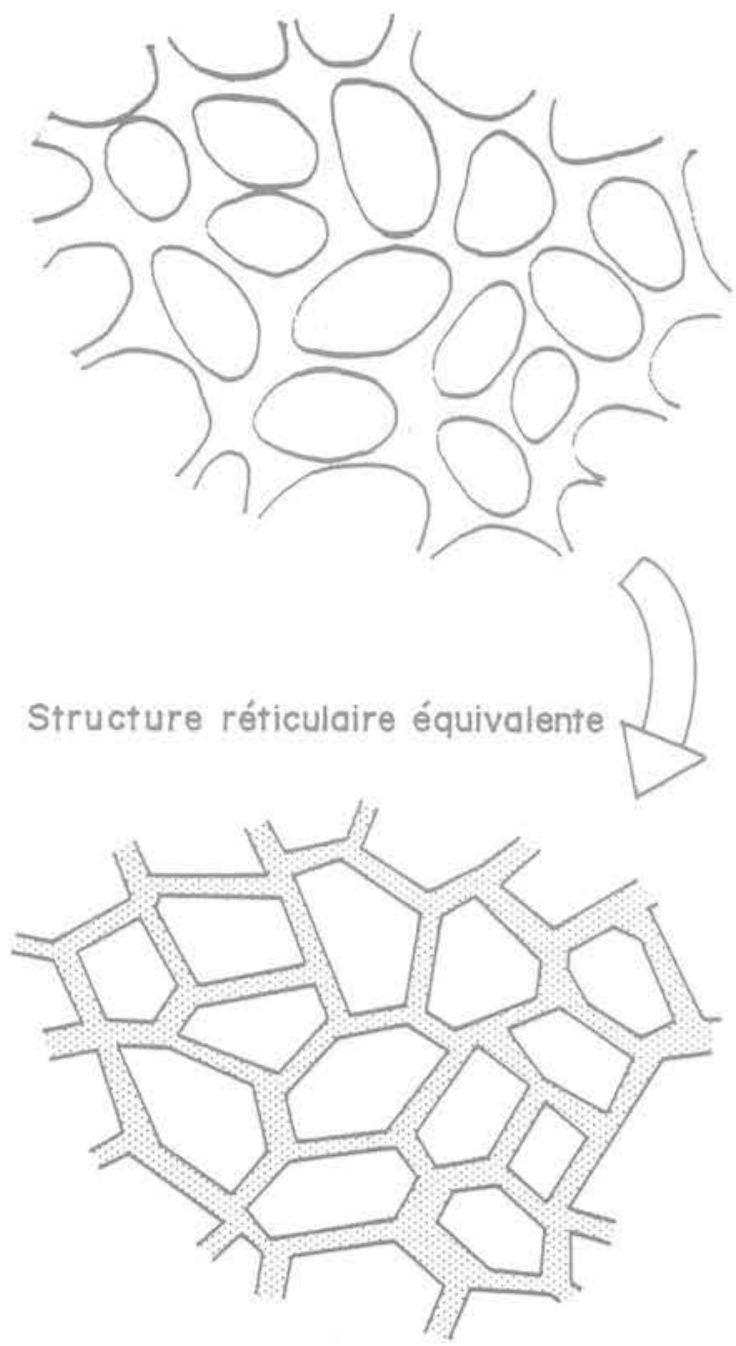

Fig. 13 Structure vacuolaire uniforme et structure réticulaire équivalente

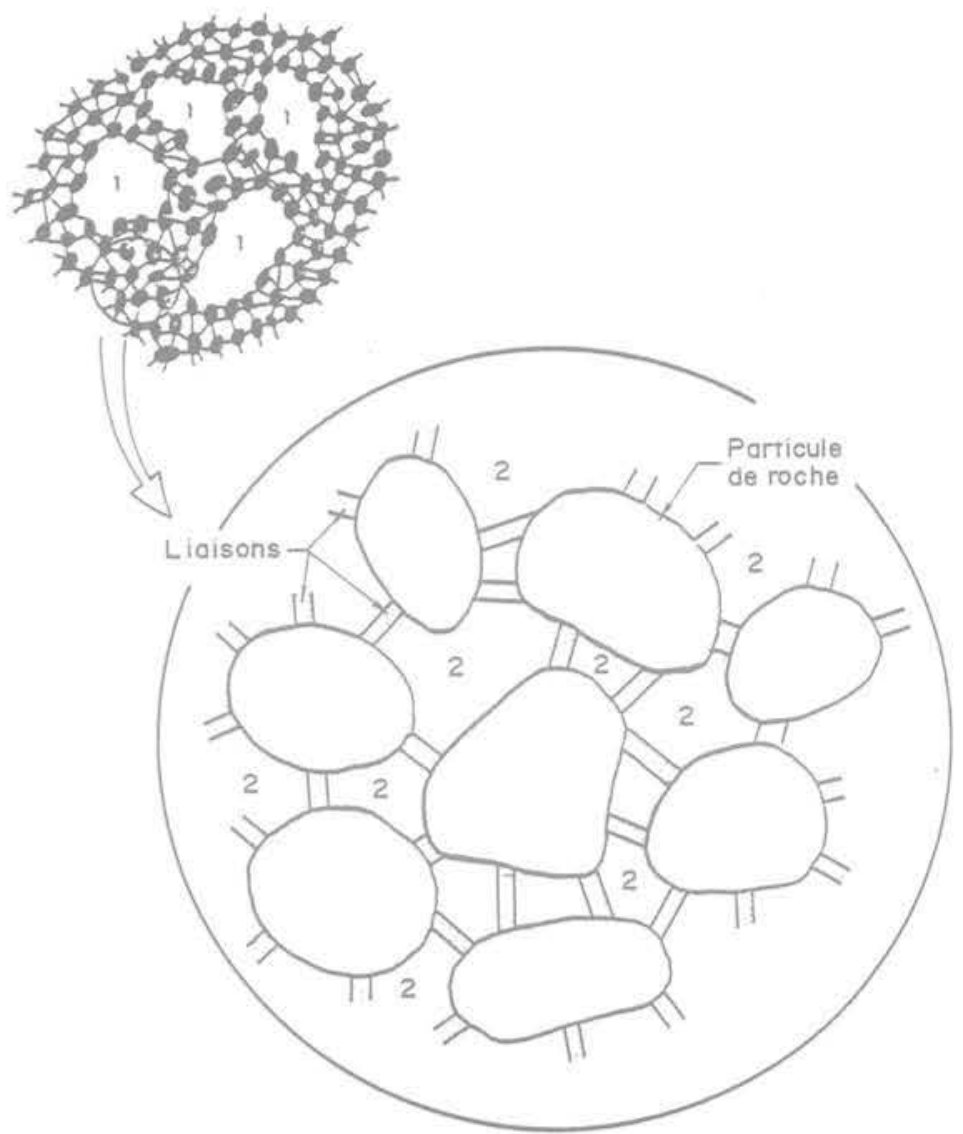

Fig. 14 Structure vacuolaire avec liaisons. Noter les deux échelles: 1 grands vides, 2 petits vides

\section{Interprétation théorique}

Afin d'interpréter théoriquement les comportements observés, il faut inévitablement schématiser les structures réelles qui sont trop complexes. Une structure vacuolaire uniforme peut être schématisée de la façon indiquée sur la figure 13. Le matériau qui englobe les vides peut être assimilé à un nid d'abeilles irrégulier, dans lequel le matériau solide forme une sorte de structure réticulaire. II y a quelquefois de grands vides dans cette structure uniforme. S'il s'agit de particules indépendantes, unies par des liaisons plus ou moins longues, la structure peut être assimilée au schéma de la figure 14 qui comporte aussi des grands vides et des petits.

La théorie exposée ci-dessous représente au moins de manière qualitative les comportements obtenus expérimentalement. Elle s'applique à une structure avec des vides de taille uniforme et comporte les hypothèses suivantes

a) le sol s'effondre quand les liaisons sont détruites:

b) la roche intacte est élastique jusqu'à l'effondrement, avec un module élastique $\mathrm{E}$ et un coefficient de Poisson $\mu$;

c) les liaisons ont les dimensions moyennes suivantes : largeur I, longueur L;

d) si la roche intacte est soumise à des contraintes principales $\sigma_{1} \sigma_{3}$ et si les déformations sont $\varepsilon_{1} \varepsilon_{3}$, chacune des extrémités des liaisons se déforme de $K \varepsilon_{1} K \varepsilon_{3}$, le coefficient $K$ représentant la rigidité relative des particules et des liaisons, sans rotation relative des nœuds;

e) Les liaisons sont constituées d'un matériau rocheux de module $E_{m}$ dont les résistances à la compression et la traction simples sont $\sigma_{c}$ et $\sigma_{T}$. 
Les déplacements et les contraintes dépendent de l'orientation de chaque liaison par rapport aux contraintes principales (fig. 15). Avec les hypothèses de base ci-dessus, on détermine la direction des liaisons qui se fracturent les premières. Les relations entre les contraintes principales pour arriver à cette rupture sont indiquées sur le diagramme $p, q$ de la figure 16. Deux lignes marquent le début de la rupture. l'une correspond à la rupture des liaisons par traction, l'autre par compression. Ces deux lignes correspondent respectivement à la rupture fragile et à l'effondrement. L'effondrement est par conséquent produit par une rupture des liaisons en compression.

Jusqu'à maintenant, les vides entre les particules ont été supposés de dimension uniforme. S'il y a de grands vides, la rupture se produit plus tôt par l'effet des concentrations de contraintes sur leurs bords. Dans ce cas, l'analyse théorique en supposant les vides elliptiques (fig. 17) montre qu'il y a encore deux lignes qui limitent la rupture, la ligne RF par traction, et la ligne CC par compression, déterminées par les résistances à la traction et à la compression de la structure avec de petits vides étudiée au préalable (fig. 16).

D'après ces approches théoriques, les lignes qui définissent la rupture fragile et l'effondrement sont deux droites. Ces lois correspondent assez bien aux résultats expérimentaux. Comme la nature ne permet guère de points anguleux, les deux droites sont raccordées par une courbe conformément aux essais.

La ligne qui marque la fin de l'effondrement (ligne FC) reste mal définie d'après les essais de laboratoire ou in situ puisque la transition vers un sol pulvérulent est progressive. De même les modèles mathématiques que l'on peut adapter sont douteux. Les théories de Cambridge sur les sols granulaires permettent de retrouver ces lignes sur un diagramme de Mohr, mais les hypothèses sont variées. Si on suppose que la trajectoire des déformations suit la loi de la normalité, ces lignes sont elliptiques, avec une tangente verticale sur l'axe $p$ et une tangente horizontale au raccordement avec la ligne de rupture finale.

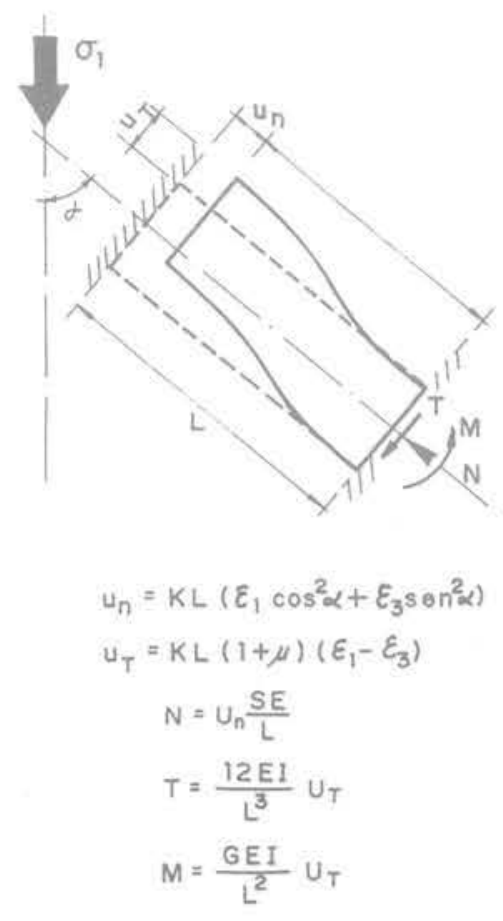

Fig. 15 Déplacements et efforts sur les liaisons

\section{Résultats géotechniques}

Les résultats obtenus sur les roches de fondation des barrages de Los Campitos et Ariñez, pour lesquels les études et expérimentations ont été les plus complètes, sont présentés de manière à mettre en évidence les lignes de rupture fragile et d'effondrement. Sur le premier site (fig. 18) la ligne d'effondrement par compression peut être assimilée à une droite faisant avec l'axe $p$ un angle d'environ $20^{\circ}$ alors que la ligne de rupture fragile fait un angle de l'ordre de $30^{\circ}$. Pour l'agglomérat d'Ariñez (fig. 19) l'angle de la ligne CC est de l'ordre de $45^{\circ}$. Cela veut dire que l'effondrement a lieu quand la contrainte principale majeure atteint une valeur constante égale à $\sigma_{c}$. Cette valeur varie entre 2,7 et $3.5 \mathrm{MPa}$. Dans les deux cas on remarque dans les essais de laboratoire que la direction principale d'effondrement est parallèle à la contrainte principale majeure avec des déformations très petites dans la direction perpendiculaire. Ce fait met en évidence que les liaisons qui se fracturent en premier lieu sont probablement celles dont la direction est parallèle à la contrainte majeure.

La figure 20 rassemblé les résultats des essais d'effondrement en compression isotrope, en fonction de la densité sèche du matériau. Ils s'ordonnent très clairement: pour les densités de l'ordre de $0.5 \mathrm{t} / \mathrm{m}^{3}$, les résistances à la compression isotrope sont très faibles, de l'ordre de $3 \mathrm{~kg} / \mathrm{cm}^{2}$; pour des densités de l'ordre de $1,6 \mathrm{t} / \mathrm{m}^{3}$, elles s'élèvent à $45 \mathrm{~kg} / \mathrm{cm}^{2}$ (respectivement 0,3 et $4,5 \mathrm{MPa}$ ).

Pour la résistance en compression simple (fig. 21) la corrélation est moins évidente; néanmoins, on constate aussi un accroissement net avec la densité jusqu'à $1.2 \mathrm{t} / \mathrm{m}^{3}$; pour des densités supérieures, les résultats sont plus erratiques et varient entre 10 et $50 \mathrm{~kg} / \mathrm{cm}^{2}(1$ et $5 \mathrm{MPa}$ ).

La figure 22 présente les modules de déformation obtenus dans les essais de laboratoire. Les zones hachurées se rapportent aux essais triaxiaux avec déviateur, et les limites marquées par des flèches aux résultats obtenus dans les essais de compression isotrope avant l'effondrement. Ils varient entre 1000 et $8000 \mathrm{~kg} / \mathrm{cm}^{2}$ (100 et $800 \mathrm{MPa}$ ).

On a effectué aussi des essais à la plaque sur une surface de $1 \times 1 \mathrm{~m}^{2}$, dont les résultats sont indiqués sur la figure 23. On observe clairement l'influence de la densité. On constate aussi que les modules de déformation in situ sont très supérieurs à ceux obtenus en laboratoire sur de petites éprouvettes. Avant l'effondrement, le module de déformation varie entre 9000 et $30000 \mathrm{~kg} / \mathrm{cm}^{2}$ (900 et $\left.3000 \mathrm{MPa}\right)$. Le comportement est donc celui d'une véritable roche.

\section{Application aux ouvrages, Interaction ouvrage-roche}

Plutôt que d'analyser le comportement de ces roches en relation avec différents types d'ouvrages de génie civil, on se bornera à étudier le cas des fondations de type superficiel : soit par exemple le cas d'une semelle rigide sur la surface de ce terrain. Deux points intéressants méritent d'être étudiés, d'une part la pression de contact qui provoque le début de l'effondrement généralisé, d'autre part les tassements prévisibles dans le cas où cette pression est dépassée.

La figure 24 montre les conditions dans lesquelles la roche commence à s'effondrer. Avant d'arriver à la pression critique, la distribution des contraintes entre 


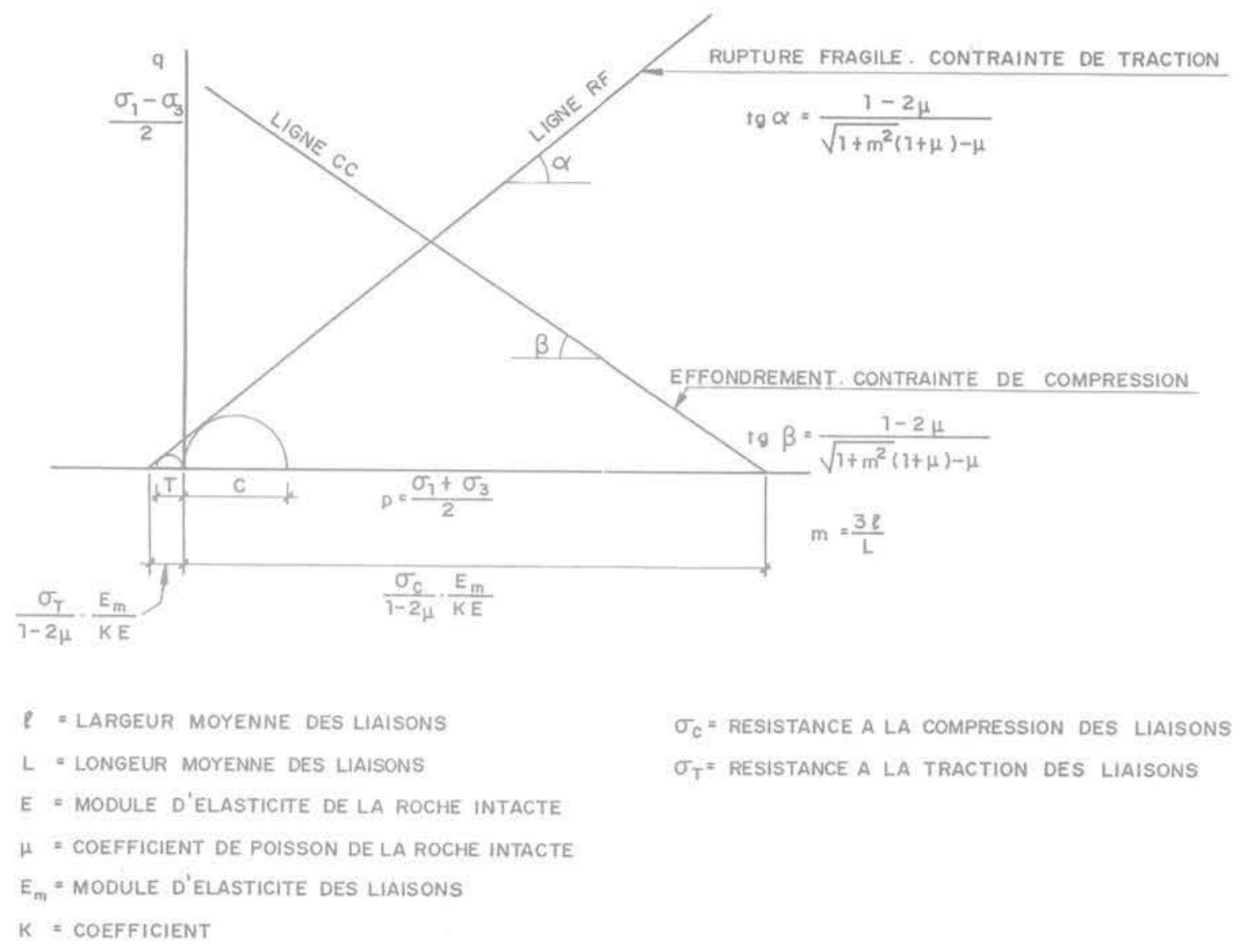

Fig. 16 Structure réticulaire avec liaisons

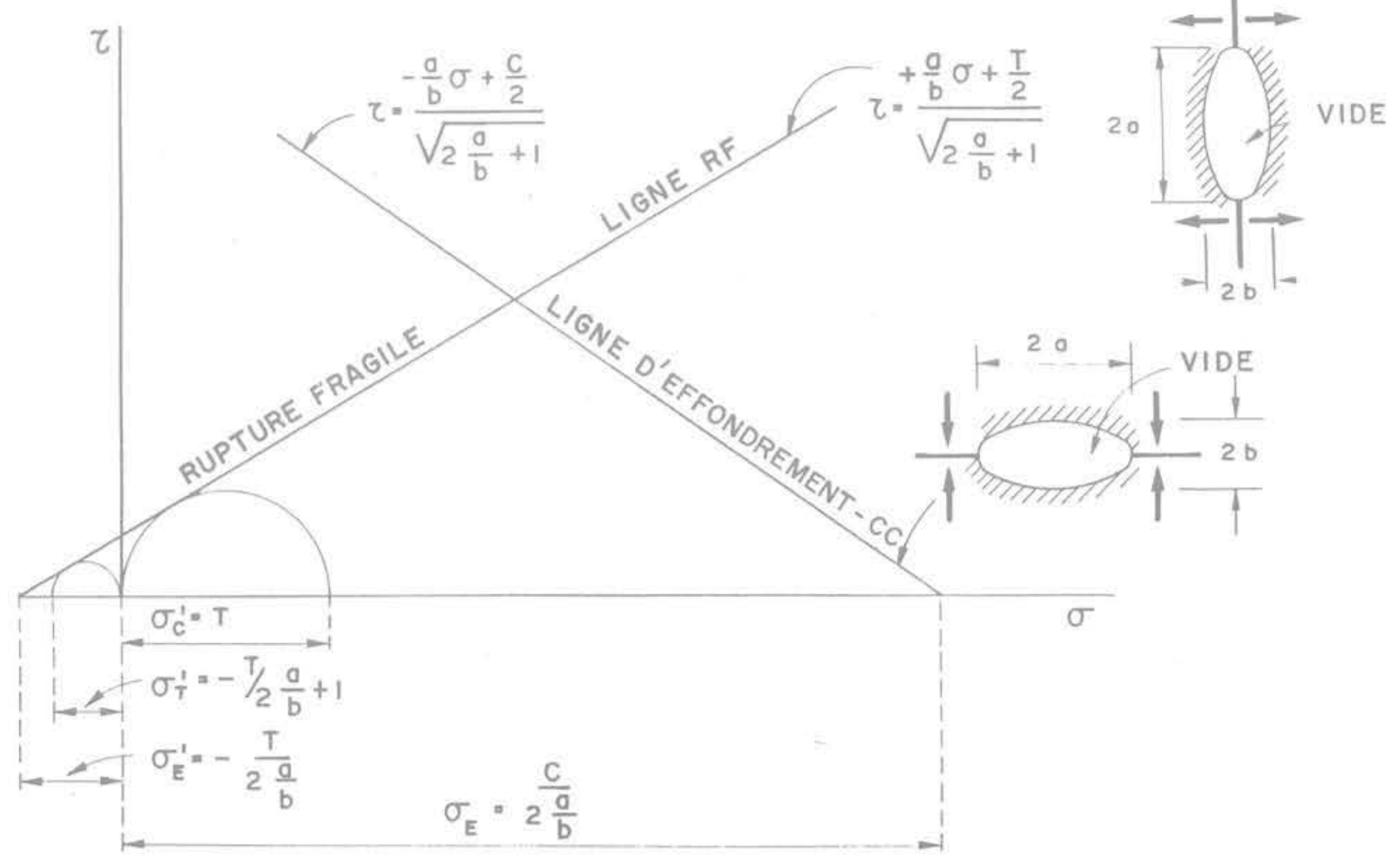

Fig. 17 Structure vacuolaire à grands vides elliptiques 


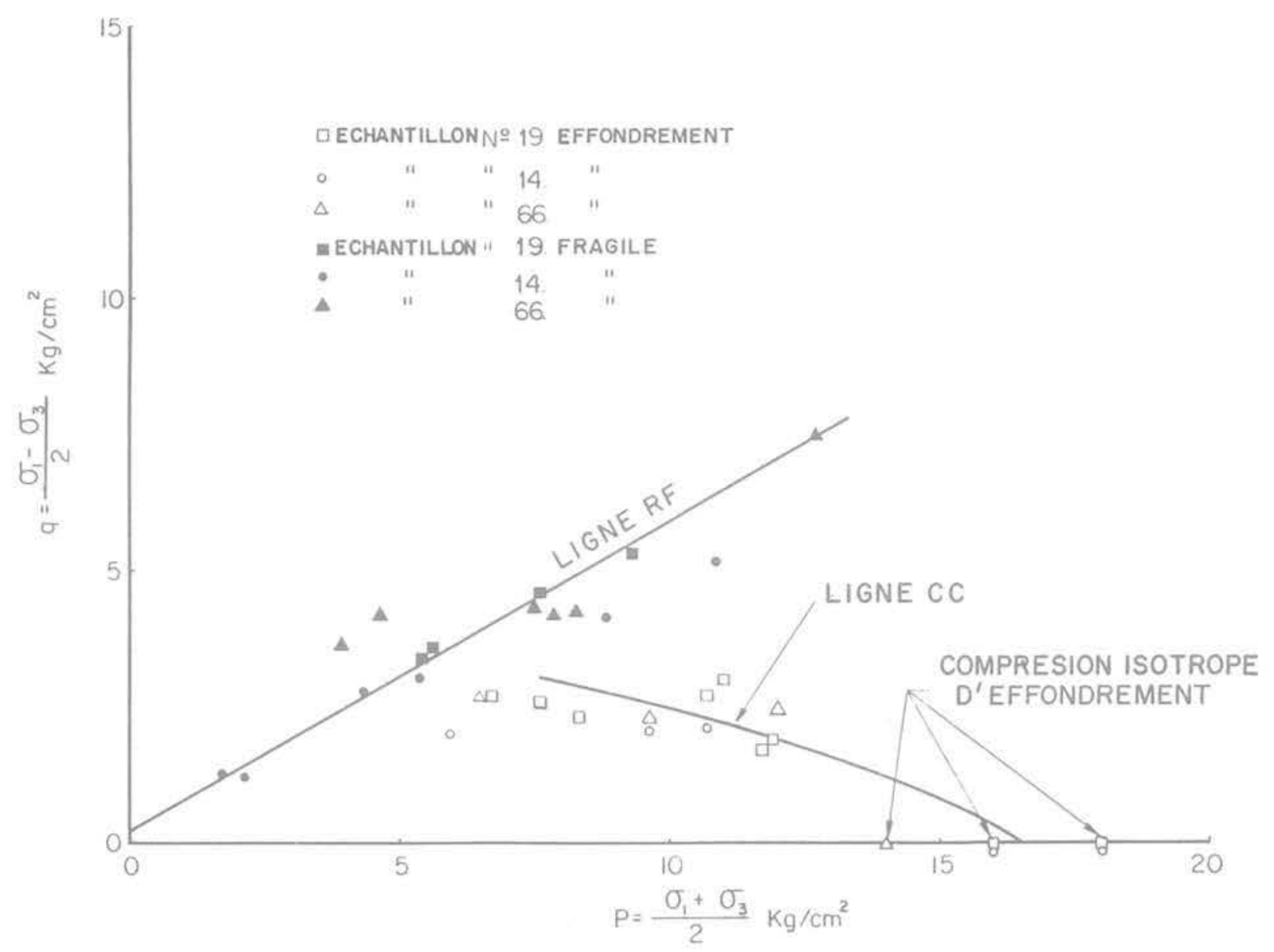

Fig. 18 Résultats des essais sur le site du barrage "Los Campitos»

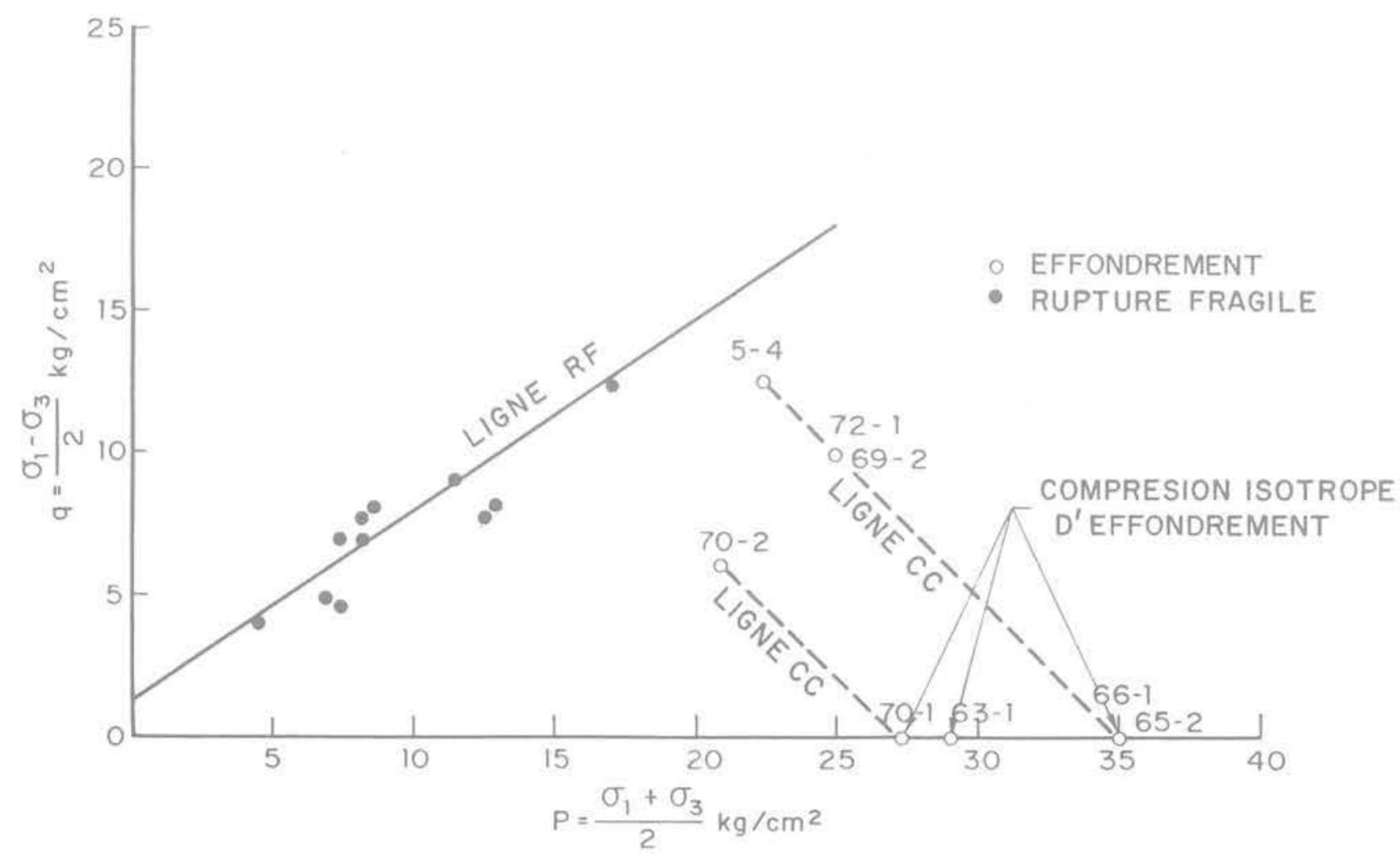

Fig. 19 Résultats des essais sur le site du barrage "Arinez» 
semelle et roche, supposée élastique, présente la configuration classique avec des valeurs très élevées sur les bords. Par conséquent, c'est ici d'abord que l'effondrement se produit. La contrainte reste limitée à la pression verticale qu'il s'agit de définir. En tenant compte de ce que le comportement semelle-roche empêche toute déformation en direction horizontale vers l'extérieur de la semelle, l'état de contrainte est pratiquement le même que celui existant au repos, c'est-à-dire $\sigma_{1}$ sur les plans horizontaux et $\mathrm{K}_{0} \sigma_{1}$ sur les plans verticaux ( $K_{0}$ étant la valeur indiquée sur la figure 24 en fonction du coefficient de Poisson de la roche non écrasée). En augmentant la pression l'effondrement se propage vers l'intérieur de la semelle, et quand il atteint le centre, la roche sous la semelle se trouve partout dans le même état de contrainte. Le cercle de Mohr doit se trouver sur la ligne de début d'effondrement (ligne CC) et d'autre part les contraintes principales doivent vérifier la relation $\sigma_{3}=K_{0} \sigma_{1}$.

La figure 24 montre le cercle de Mohr correspondant et la contrainte qui provoque l'effondrement en fonction de la contrainte isotrope critique $\sigma_{c}$ et de l'angle $\beta$ qui définissent la ligne $\mathrm{CC}$. Si la contrainte appliquée par la semelle dépasse cette valeur, la roche s'effondre progressivement en formant sous la semelle un matelas qui uniformise les contraintes. L'expérience met en évidence un phénomène essentiel : le tassement de la semelle s'accompagne du découpage de la roche suivant le périmètre de la semelle. L'effondrement ne s'étend pas au-delà de ce contour, c'est-à-dire que la semelle s'enfonce progressivement dans un trou cylindrique. Une comparaison peut éclairer ce phénomène. En effet la neige est un matériau susceptible d'effondrement et lorsqu'on marche dessus, la chaussure s'enfonce profondément en laissant une trace égale ou similaire au contour de la semelle.

Cette même circonstance s'observe dans les essais de charge exécutés in situ bien que. logiquement, l'effondrement soit beaucoup plus limité. Le matelas de roche effondrée reste emboité entre des parois verticales de roche intacte. L'état de contrainte à l'intérieur de ce matelas est complexe. On peut estimer d'une façon approximative l'épaisseur $h$ de la roche effondrée: sur les parois verticales du contour de la semelle, le matériau effondré frotte contre la roche intacte. Le cercle de Mohr est approximativement celui numéroté 2 sur la figure 25. En effet il doit être à la fois situé sur la droite intrinsèque du matériau pulvérisé et sur la ligne d'effondrement de la roche. Par ailleurs dans le fond du trou, le matériau se trouve dans des conditions similaires à celle d'un essai cedométrique. son cercle de Mohr est celui numéroté 1.

Avec ces données, la profondeur moyenne atteinte par l'effondrement sur le bord est celle indiquée sur la figure 25, A étant l'aire de la semelie ot $P$ son périmètre. Les essais ont démontrè que l'effondrement est plus profond dans la partie centrale à cause des contraintes plus élevées dans cette zone. La figure 26 indique les résultats d'un essai de charge sur une plage de $1 \times 1 \mathrm{~m}^{2}$. Les points de référence pour la mesure des déformations étaient ancrés à $15 \mathrm{~cm}$ sous la surface de la plaque. Aux points 1 et 3 situés sur les bords extérieurs, l'effondrement commence pour une pression d'environ $30 \mathrm{~kg} / \mathrm{cm}^{2}$, alors qu'au centre il commence vers $25 \mathrm{~kg} / \mathrm{cm}^{2}$ (respectivement 3 et 2,5 $\mathrm{MPa}$ ).

Les critères qu'il y a lieu d'appliquer pour des fondations superficielles sur ces types de roches ne sont pas clairement fixés. Ils sont très variabies selon le

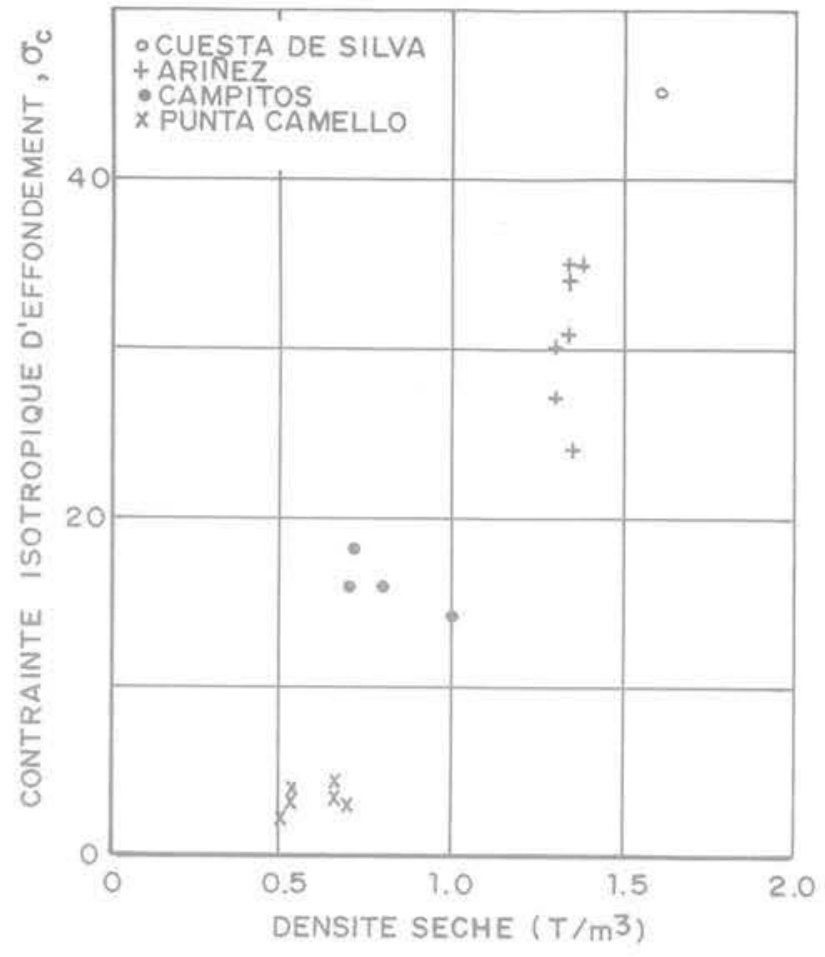

Fig. 20 Ensemble des résultats d'essais sur les quatre sites, contrainte isotrope d'effondrement en fonction de la densité

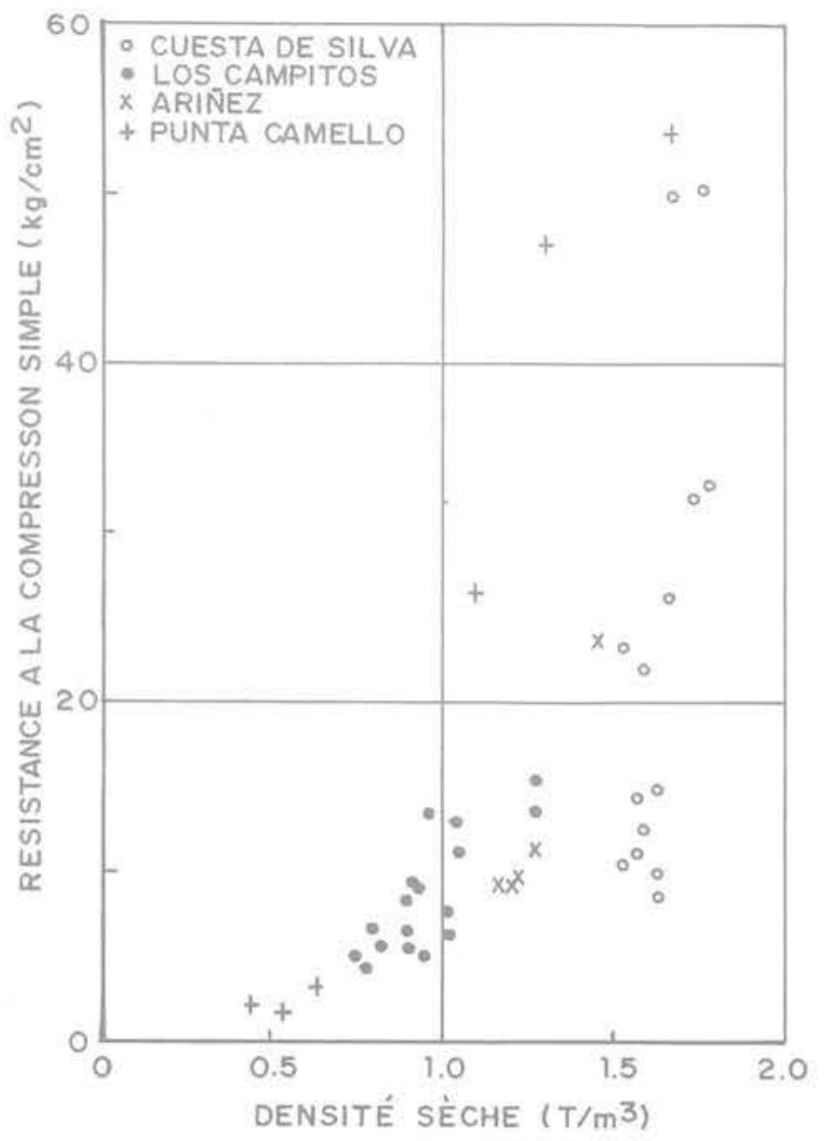

Fig. 21 Ensemble des résultats d'essais sur les quatre sites, compression simple en fonction de la densité 


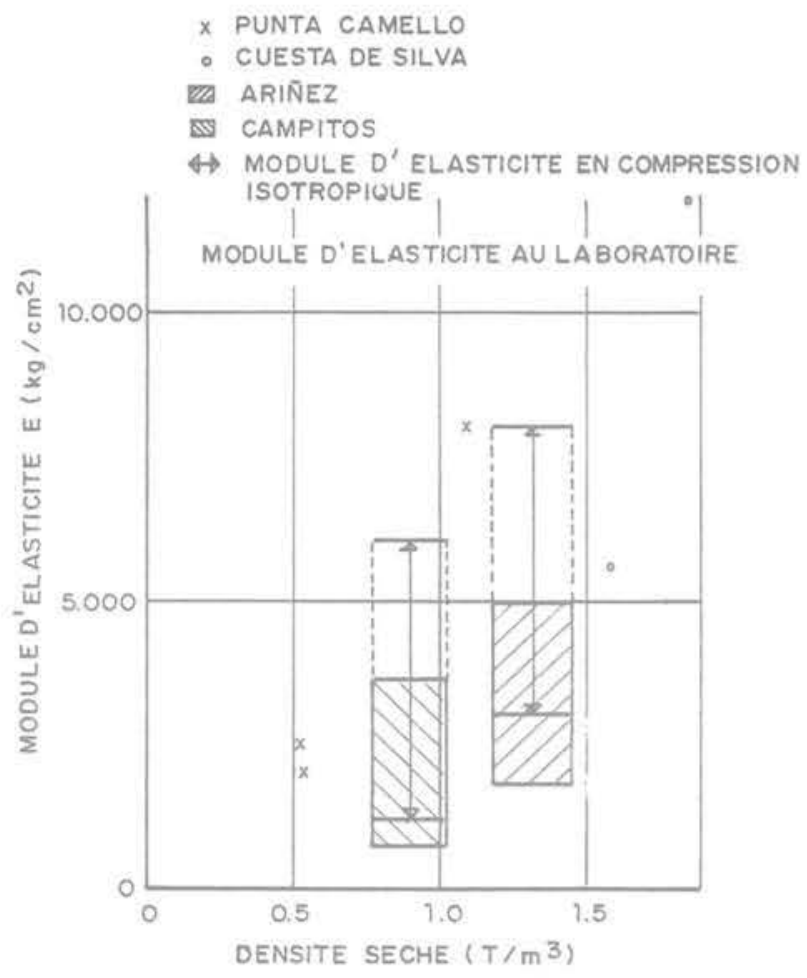

Fig. 22 Ensemble des résultats d'essais sur les quatre sites, module d'élasticité au laboratoire en fonction de la densité

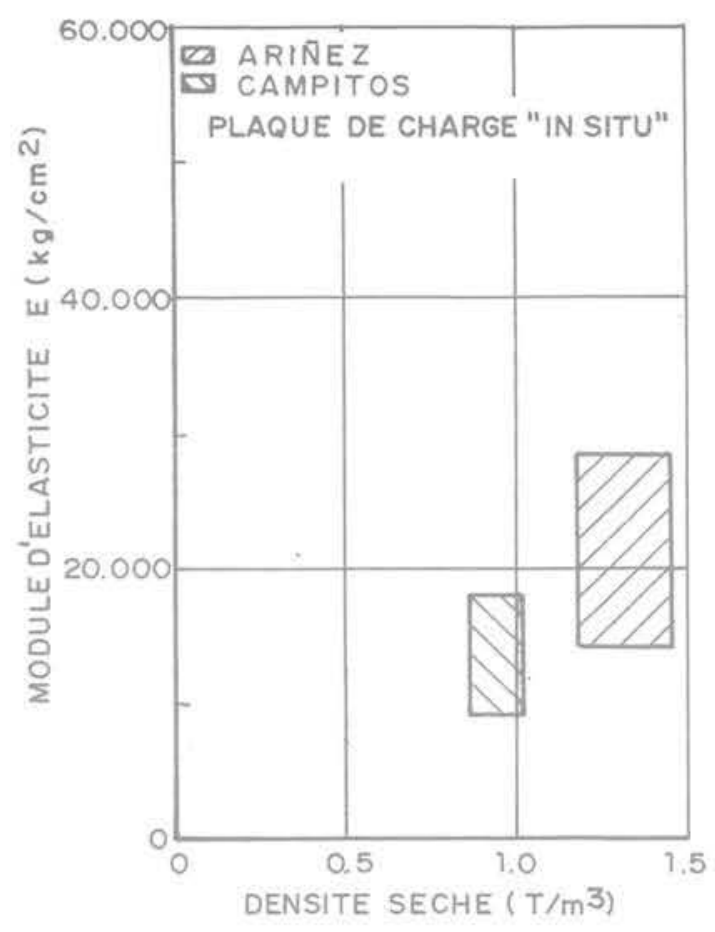

Fig. 23 Ensemble des résultats d'essais sur deux sites, module d'élasticité à la plaque de charge en fonction de la densité

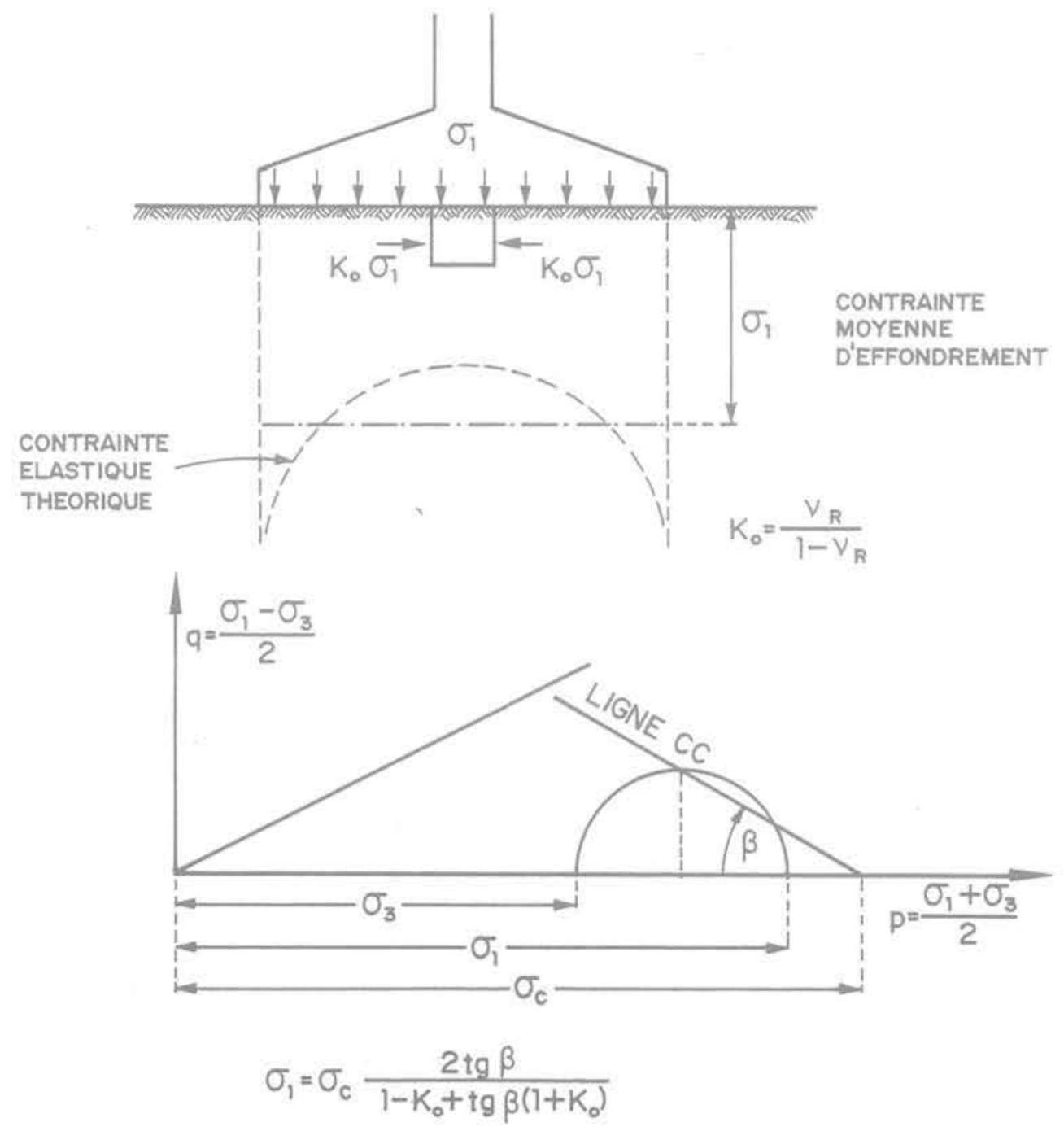

Fig. 24 Contrainte d'effondrement sous une semelle rigide 

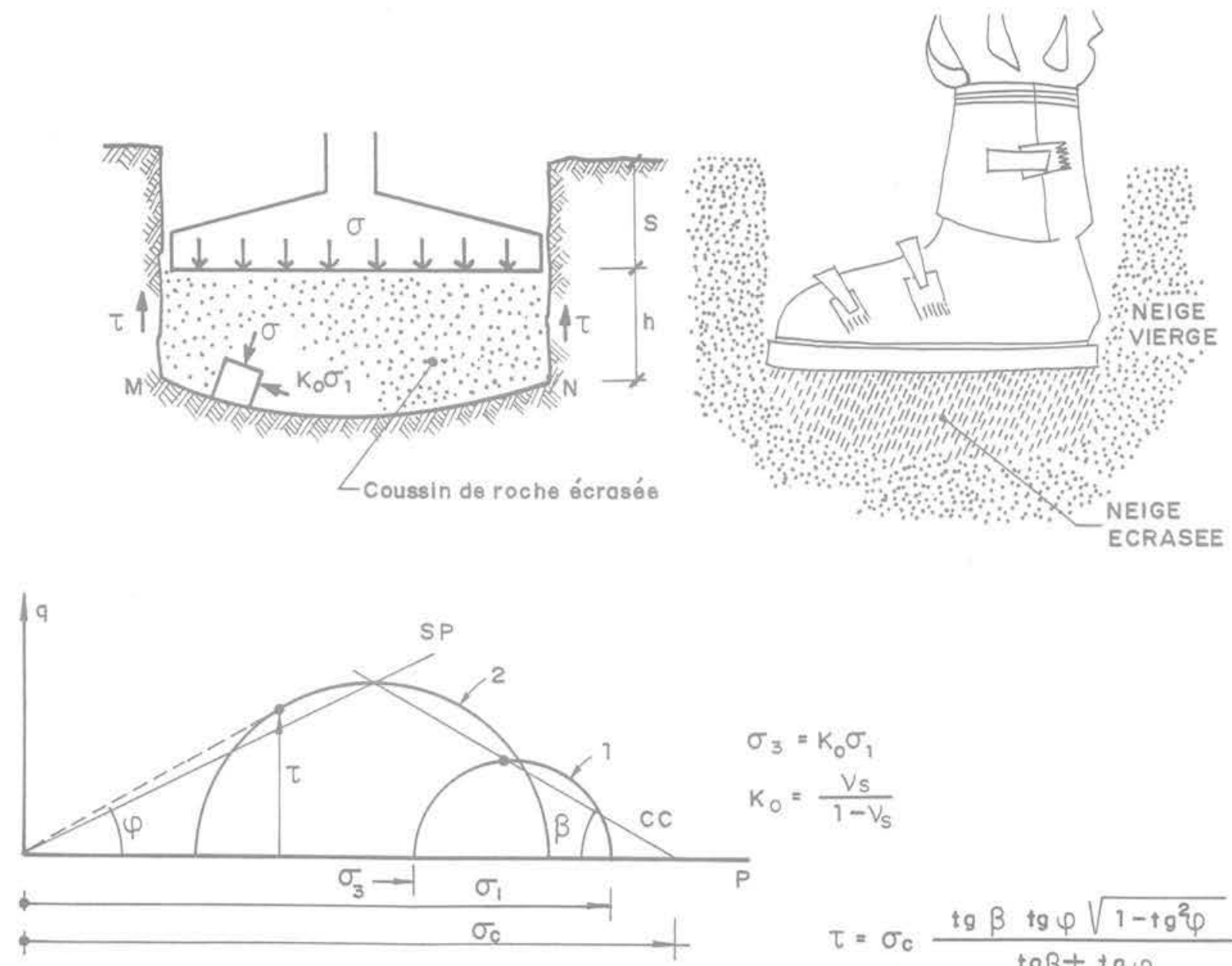

$$
\begin{aligned}
& \sigma_{3}=k_{0} \sigma_{1} \\
& k_{0}=\frac{V_{s}}{1-V_{s}} \\
& \tau=\sigma_{c} \frac{\operatorname{tg} \beta \operatorname{tg} \varphi \sqrt{1-t^{2} \varphi}}{\operatorname{tg} \beta+\operatorname{tg} \varphi} \\
& h=\left(\sigma-\sigma_{1}\right) \frac{A}{P \tau}
\end{aligned}
$$

$A=$ Surfoce de la semelle

$P=P e ́ r l m e ̀ t r e d e l a s e m e l l e ~$

Fig. 25 Tassement d'une semelle rigide et analogie avec l'enfoncement de la chaussure du skieur dans la neige vierge

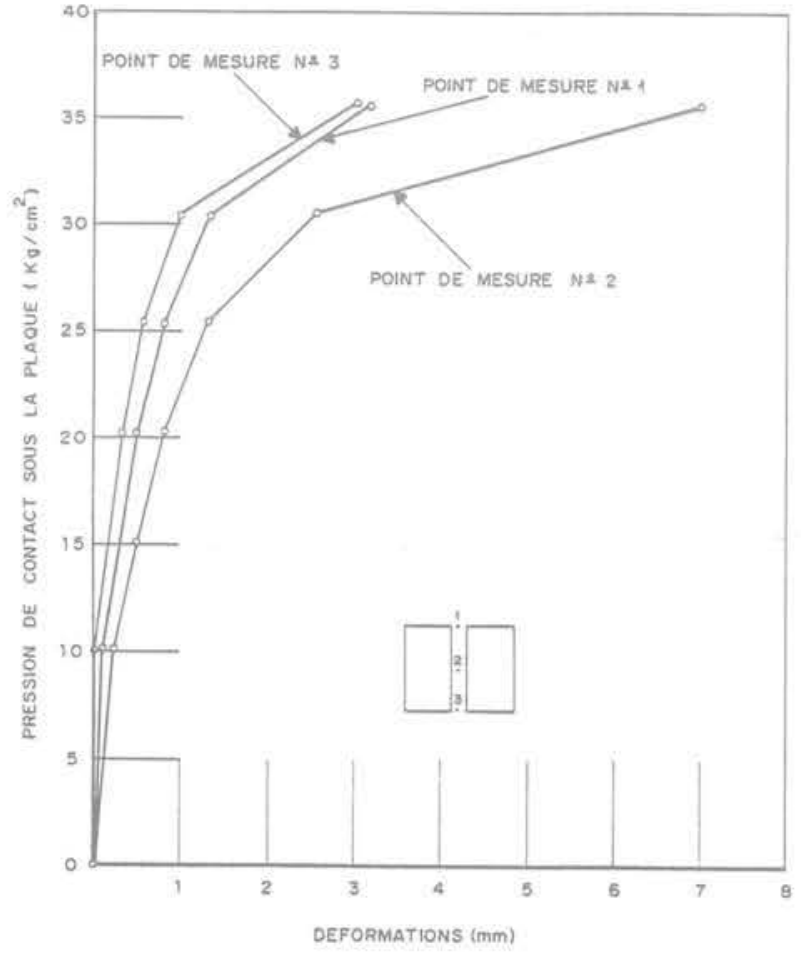

Fig. 26 Essai de chargement in situ avec une plaque de 1 mètre carré au barrage de "Los Campitos»

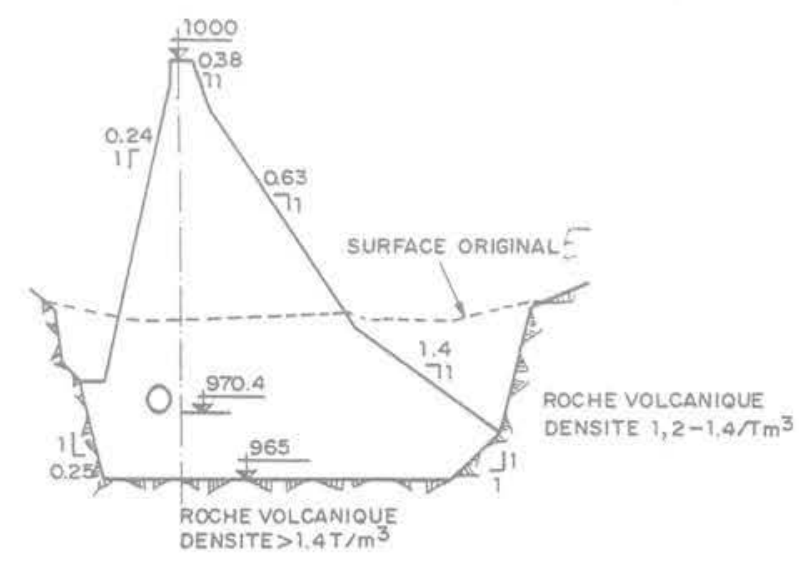

Fig. 27 Coupe transversale schématique du barrage "Arinez» montrant l'importance des fouilles en déblai pour atteindre une roche de densité supérieure à $1,4 \mathrm{t} / \mathrm{m}^{3}$ 


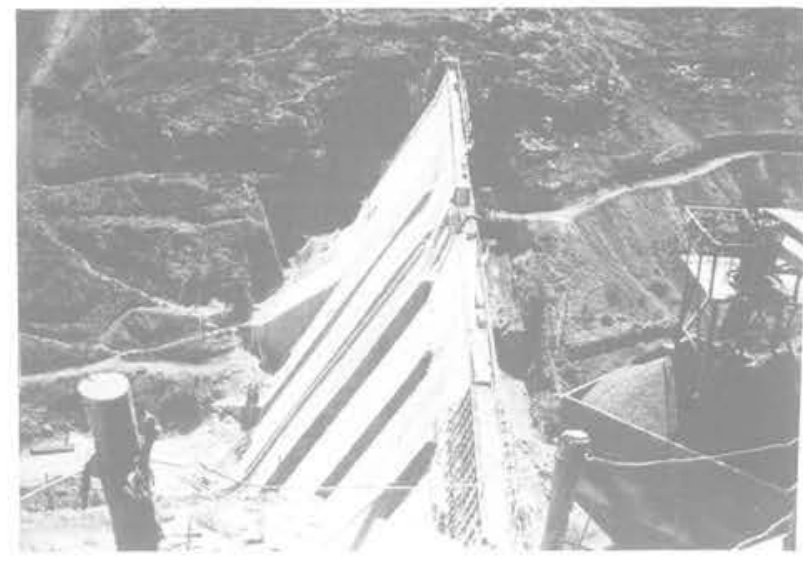

Fig. 28 Photographie du barrage "Arinez» : la fouille et l'élargissement de la section en rive droite sont bien visibles

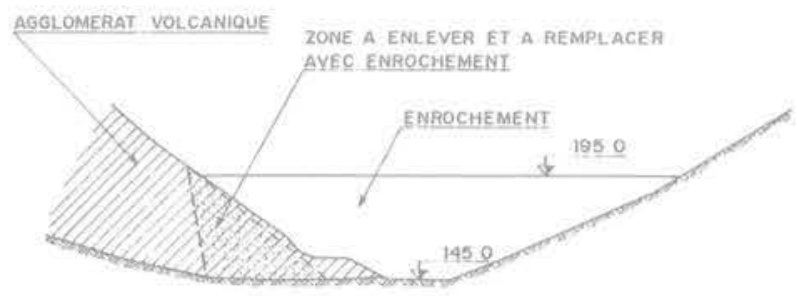

Fig. 29 Coupe longitudinale schématique du barrage "Los Campitos" montrant le déblai remplacé par l'enrochement

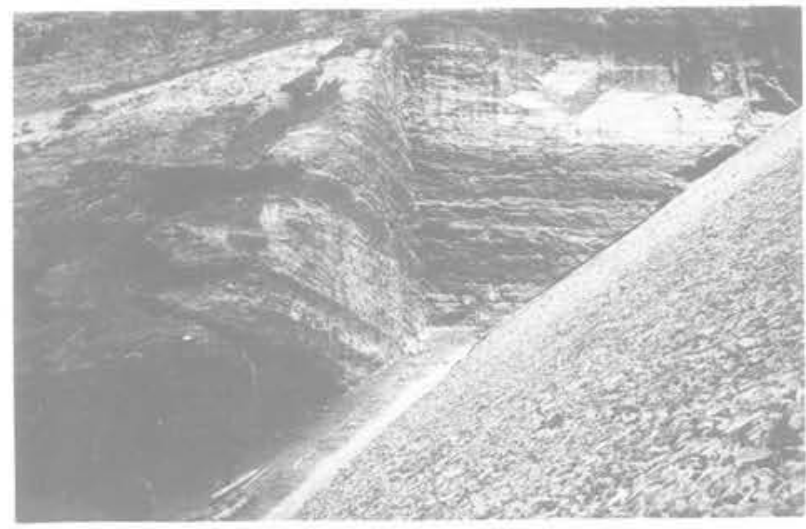

Fig. 30 Barrage "Los Campitos", photographie de la fouille dans l'agglomérat de la rive droite

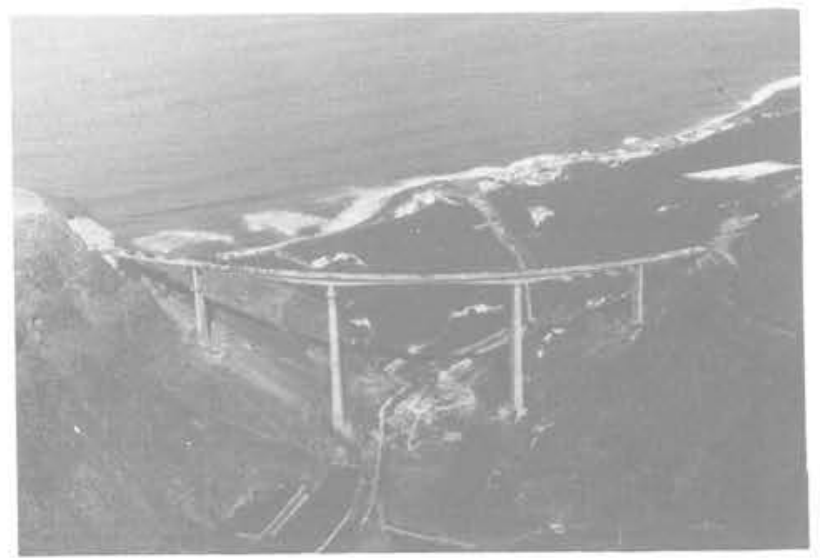

Fig. 31 Viaduc de Costa de Silva degré d'effondrement de l'agglomérat. Théoriquement, on pourrait admettre des contraintes supérieures à celles qui provoquent l'effondrement sous la semelle, tant que les tassements finaux restent dans la limite des tassements admissibles pour les structures à construire. Ceci peut être le cas d'ouvrages peu importants.

Par contre pour des ouvrages exigeant un haut niveau de sécurité, il faut se situer du côté de la sécurité par rapport au phénomène d'effondrement. Si celui-ci se produit lors des étapes finales de la construction, quand les contraintes sur le terrain sont importantes, les tassements peuvent affecter l'ensemble de l'ouvrage et produire, suivant le type de barrage, soit des fissures dans le couronnement des barrages en terre, soit des mouvements entre les plots des barrages poids. Par conséquent les critères appliqués jusqu'à maintenant sont du côté de la sécurité, et on tend à spécifier que la pression maximale du barrage sur un agglomérat susceptible d'effondrement doit être inférieure, avec un coefficient de sécurité de 5 , à celle qui provoquerait le début de l'effondrement.

C'est le cas pour le barrage d'Ariñez, sur son appui rive droite. II s'agit d'un barrage à contreforts, dont la partie centrale et la culée gauche sont fondées sur des formations volcaniques typiques de l'île de Grande Canarie, appelée "Roque Nublo". Mais dans l'appui droit, il y a des agglomérats effondrables qui ont fait l'objet d'essais exhaustifs (et dont les résultats ont été exposés ci-dessus). Dans la partie haute de l'appui, la roche présente des densités faibles, de l'ordre de $1.2 \mathrm{t} / \mathrm{m}^{3}$, qui augmentent progressivement avec la profondeur jusqu'à atteindre $1,6 \mathrm{t} / \mathrm{m}^{3}$.

Les contraintes critiques d'effondrement varient entre 25 et $35 \mathrm{~kg} / \mathrm{cm}^{2}$ (2,5 et $3,5 \mathrm{MPa}$ ). II a fallu spécifier que le versant devrait être excavé jusqu'à ce que la densité minimum de la roche d'appui dépasse 1,4 , et que la contrainte maximale exercée par le barrage ne dépasse pas $7 \mathrm{~kg} / \mathrm{cm}^{2}$ (d'après le critère déjà exposé).

Sur la figure 27 , on remarque le profil adopté pour le barrage pour remplir cette condition (le barrage achevé sur la figure 28). On peut noter un accroissement de l'excavation dans l'appui rive droite pour satisfaire aux conditions indiquées. Cet ouvrage est probablement un record mondial par la densité de la roche de fondation d'un barrage $\left(1,4 \mathrm{~kg} / \mathrm{cm}^{3}\right)$. Sa retenue est remplie et son comportement est normal.

Pour le barrage de Los Campitos (fig. 29) de hauteur $60 \mathrm{~m}$, la décision a été différente. Sur l'appui rive droite où se trouvent les agglomérats susceptibles d'effondrement, les pressions exercées par l'ouvrage, bien qu'insuffisantes pour provoquer l'effondrement, ne laissaient pas un coefficient de sécurité suffisant. C'est pourquoi une partie de l'appui a été excavée et remplacée par des enrochements (fig. 30 ). La retenue n'a pas pu être remplie à cause d'importantes fuites au large du barrage. II n'est par conséquent pas possible pour le moment de juger le comportement global de l'ouvrage lui-même. II ne devrait pas exister d'importants problèmes liés à l'effondrement du terrain.

La figure 31 montre le pont de Costa de Silva, fondé sur les agglomérats décrits antérieurement. Les piles sont fondées sur des puits de diamètre $1,5 \mathrm{~m}$ et de profondeur environ $15 \mathrm{~m}$. creusés à la main.

Enfin si l'épaisseur de roche effondrable est grande, une solution du problème pourrait consister à provoquer l'effondrement par impact avant de construire l'ouvrage. La consolidation dynamique (Ménard) serait une solution bien qu'il n'y ait aucune référence comparable. 
Dans les excavations de talus pour les routes, aucun problème important n'a été signalé. La roche est facile à excaver et les contraintes dans les talus ne sont pas suffisamment élevées pour provoquer l'effondrement.

\section{Remerciements :}

L'auteur exprime ses remerciements au professeur J.A. Jimenez Salas et au professeur A. Serrano Gonzalez pour leur collaboration et pour l'autorisation de publier ces résultats. 